Topiques, études satoriennes

Topoï Studies, Journal of the SATOR

\title{
Clément Marot élève de Jean : le modèle des récits de formation allégoriques
}

\section{Ellen Delvallée}

Volume 4, 2018

Maître-disciple : une relation topique

URI : https://id.erudit.org/iderudit/1074718ar

DOI : https://doi.org/10.7202/1074718ar

Aller au sommaire du numéro

Éditeur(s)

SATOR, Société d'Analyse de la Topique Romanesque d'Ancien Régime

ISSN

2369-4831 (numérique)

Découvrir la revue

Citer cet article

Delvallée, E. (2018). Clément Marot élève de Jean : le modèle des récits de formation allégoriques. Topiques, études satoriennes / Topoï Studies, Journal of the SATOR, 4, 1-17. https://doi.org/10.7202/1074718ar
Résumé de l'article

Lorsque Clément Marot raconte sa formation au métier d'écrivain auprès de son père, dans L'Enfer, l'épître " Au Roy » de La Suite et L'Eglogue au Roy, soubs les noms de Pan, \& Robin, son récit se fait souvent fictif. Ces entorses à la vérité autobiographique correspondent en fait à la réécriture de topoï issus des récits de formation allégoriques. Marot mobilise ces topoï à des fins rhétoriques - lorsqu'il se défend ou réclame des biens - mais aussi esthétiques - car ils servent au poète à redéfinir son art et l'exercice même du métier d'écrivain courtisan. 


\section{Clément Marot élève de Jean : le modèle des récits de formation allégoriques}

Dans différents poèmes composés tout au long de sa carrière, Clément fait le récit de son enfance et de sa formation au métier d'écrivain auprès de Jean, son père, également poète de François $\mathrm{I}^{\mathrm{er}}$, et auquel Clément a succédé à sa mort. Toutefois, la critique a depuis longtemps remarqué que ce récit autobiographique est en maints endroits fictif, invitant à la prudence quiconque désirant reconstituer la vie de l'aspirant écrivain courtisan sur la foi de ses poèmes ${ }^{1}$. Ces récits, présents dans L'Enfer, l'épître " Au Roy » de La Suite de l'Adolescence clémentine et l'Eglogue au Roy, soubs les noms de Pan, \& Robin ${ }^{2}$ sont en effet idéalisés, chronologiquement incohérents et parfois même écrits à partir de sources uniquement littéraires. Plutôt que de mesurer combien le poète s'écarte de la réalité en manipulant ou en masquant ses biographèmes, nous proposons ici d'étudier l'insertion de ces éléments biographiques dans une trame narrative codifiée, celle d'un récit de formation allégorique, ou d'un pèlerinage retraçant le parcours poétique exemplaire du disciple Marot, élevé (conformément aux topoï du genre) dans une nature idyllique avant d'être confronté à un parcours initiatique semé d'obstacles symboliques, qu'il ne traverse, pour sa gloire ou son salut, qu'à l'aide du guide bienveillant qu'est son père Jean ${ }^{3}$.

Philippe Maupeu a consacré sa thèse ${ }^{4}$ à l'étude des rapports entre «autobiographie et allégorie narrative » dans les pèlerinages des $\mathrm{XIV}^{\mathrm{e}}$ et $\mathrm{XV}^{\mathrm{e}}$ siècles. Il existe en effet une tradition de pèlerinages initiatiques et moraux dont l'exemple le plus célèbre est celui de Guillaume de Deguileville (ou Digulleville), auteur au XIV ${ }^{\mathrm{e}}$ siècle du Pèlerinage de vie humaine, dans lequel un moine de Chaalis (comme Deguileville) voit Jérusalem en vision et

\footnotetext{
${ }^{1}$ Voir l'article de Gérard Defaux, «Clément Marot: poésie, autobiographie et roman », dans Writing the Renaissance, 1992, p. 79-91. Plus récemment, Guillaume Berthon a insisté sur cette nécessaire prudence dans "Clément Marot et la tentation biographique», dans Cahiers parisiens, 2008, p. 541-551, ainsi que dans L'Intention du poète. Clément Marot « autheur », 2014.

${ }^{2}$ Clément Marot, Euvres poétiques, 2 tomes, 1990 et 1993, abrégés en $O P 1$ et $O P 2$.

${ }^{3}$ La façon dont Marot raconte sa vie et recueille ses œuvres a déjà été rapprochée de la structure d'un pèlerinage par Evelyne Berriot-Salvadore ("Le "pèlerinage de vie humaine" de Clément Marot ", Revue de littératures françaises et comparée, 7, 1996, p. 47-53) : elle explique que les voyages, refuges, ou errances, chez Marot, ont toujours un sens d'édification religieuse, et rapprochent le poète de Dieu. Nous envisagerons ici plutôt les fonctions rhétoriques et esthétiques du recours à ce genre par Clément, plus précisément dans les poèmes où il évoque Jean.

${ }^{4}$ Philippe Maupeu, Pèlerins de vie humaine. Autobiographie et allégorie narrative, de Guillaume de Deguileville à Octovien de Saint-Gelais, 2009.
} 
veut s'y rendre. Le Chemin de long estude, Le Livre de Mutacion de Fortune et Le Livre de l'advision Cristine, écrits par Christine de Pizan, nous intéressent aussi particulièrement en ce que ces pèlerinages sont moins religieux que politiques et poétiques, à l'instar des compositions du fils Marot ${ }^{5}$. Enfin, Octovien de Saint-Gelais a rédigé un Séjour d'Honneur dans lequel il raconte son arrivée à l'ermitage d'Entendement, après avoir suivi les mauvais conseils de Sensualité dans sa jeunesse. Le nombre important de manuscrits médiévaux présentant ces textes ainsi que l'actualité de leurs éditions au $\mathrm{XVI}^{\mathrm{e}}$ siècle ${ }^{6}$ témoignent de l'influence de ce genre littéraire ${ }^{7}$. L'alliance paradoxale entre allégorie et autobiographie « offre chez l'écrivain des $14^{\mathrm{e}}$ et $15^{\mathrm{e}}$ siècles une manière privilégiée de se positionner, au sein du monde et de l'écriture, en tant que sujet», explique Maupeu ${ }^{8}$. Nous voudrions mettre sa démarche et ses conclusions à l'épreuve des poèmes pseudo-autobiographiques de Clément où figure son père Jean, tantôt guide bienveillant et tantôt maître en poésie. En étudiant successivement L'Enfer, l'épître « Au Roy » (XXIV) de La Suite et L'Eglogue au Roy, soubs les noms de Pan, \& Robin d'après le modèle narratif du récit de formation allégorique, nous chercherons à comprendre par quels procédés les biographèmes de Clément sont élevés au rang de topoï signifiants et surtout à quelles fins rhétoriques et esthétiques il coule le récit de son apprentissage auprès de Jean dans un tel moule.

En 1526, Clément est emprisonné au Châtelet, probablement pour avoir mangé le lard en Carême. Il écrit alors L'Enfer, vaste pièce allégorique dans laquelle il dépeint à partir de Dante et de Virgile l'horreur du milieu judiciaire qu'il découvre, où, en dépit du droit et à cause de la cupidité des juges et avocats, les innocents comme lui sont les premières victimes. Le poète narre sa descente aux Enfers après l'accusation de l'inconstante Luna, décrit les êtres fabuleux qu'il croise et tire les conclusions morales de sa catabase judiciaire, conformément au genre du pèlerinage médiéval, défini par Siegfried Wenzel dans « The Pilgrimage of Life

5 Contrairement aux clercs ou aux hommes, Christine de Pizan doit établir son autorité à parler. Dans Le Livre de Mutacion de Fortune, elle commence ainsi par établir sa figure auctoriale marquée par la Fortune avant de procéder au récit historique proprement dit. Comme chez Marot, on le verra, le récit de la naissance de la vocation d'écrivain se lit dans la saisie allégorique des éléments biographiques.

6 Voir Géraldine Veysseyre avec l'assistance de Julie Dobrinsky et d'Émilie Fringer, « Liste des manuscrits des trois Pèlerinages », Guillaume de Digulleville. Les Pèlerinages allégoriques, 2008, p. 425-453. L'imprimeur parisien Vérard a édité cette œuvre dès 1499, puis en 1511 et 1521. Les pèlerinages de Christine de Pizan, en revanche, ne sont, à notre connaissance, guère édités dans la première moitié du XVI $\mathrm{XI}^{\mathrm{e}}$ siècle, mais le nombre de manuscrits de ses œuvres, ainsi que le succès de sa Cité des Dames et des épîtres du débat sur le Roman de la Rose laissent supposer que ses ouvrages ne devaient pas être inconnus de Marot. Saint-Gelais est quant à lui imprimé dès 1503 par Antoine Vérard à Paris, puis par sa veuve Germaine Guyart en 1519 et quelques mois plus tard par la veuve Trepperel. La concurrence de ces éditions, comme pour Deguileville, suggère le succès de cet auteur, également poète de cour.

7 Voir Françoise Bourgeois, Stéphanie Le Briz-Orgeur, Ingrid Biesheuvel et Juliette Dor, section « Réécritures et postérité du modèle des Pèlerinages », dans Guillaume de Digulleville, Les Pèlerinages allégoriques, 2008, p. 351-423. Voir aussi l'introduction de Frédéric Duval au Séjour d'Honneur d'Octovien de Saint-Gelais, 2002. 8 P. Maupeu, Pèlerins de vie humaine, op. cit., p. 9. 
as a Late Medieval Genre ${ }^{9} »$. Mais le fonctionnement de cette allégorie est trouble, tandis que les éléments autobiographiques que Clément introduit (ses nom et prénom, ainsi que le récit de son enfance quercinoise) débordent vers la fiction. Par exemple, l'absence de songe encadrant - motif caractéristique de l'allégorie, bien que non indispensable à sa définition brouille la frontière entre figuration référentielle (même satirique) et allégorique, en ce qu'elle prive le texte d'un seuil évident ${ }^{10}$. En retour, les biographèmes de Clément sont teintés de références littéraires, de sorte que leur opposition avec le monde allégorique de L'Enfer s'en trouve réduite. «Clément» et «Marot» ne sont pas seulement des informations d'état civil, mais débordent vers des comparaisons historiques ou littéraires porteuses de qualités. De même, l'évocation du Quercy de l'enfance de Marot semble moins être une réminiscence personnelle qu'un souvenir de lecture, puisque Jean Frappier ${ }^{11}$ a bien remarqué ce que ces vers doivent à la seconde Epistre de l'Amant vert de Jean Lemaire de Belges. Tout en relevant des intertextes précis, comme celui qui unit le vers 379 de Marot (« Où le Soleil non trop excessif est ») aux vers 333-334 de Lemaire ${ }^{12}$ (« Mais le soleil, combien qu'il y fut hault, / N’y estoit point excessif ne trop chault »), Frappier souligne aussi que la présence de la seconde Epistre de l'Amant vert dans L'Enfer est aussi plus générale et structurelle. Les espaces allégoriques, poétiques et biographiques s'entremêlent. Quel sens faut-il prêter à cette porosité du poétique et de l'allégorique à la biographie, lorsque Clément évoque son enfance de poète auprès de Jean ?

Ce récit du disciple Marot ne se veut pas seulement divertissant ou lyrique, mais a un but rhétorique : la défense contre les accusations. Dans son étude, Maupeu ${ }^{13}$ rappelle que dans un chapitre du Convivio (I, 2), Dante rejette l'autobiographie comme suspecte : relevant du seul registre démonstratif, le récit de soi sera soit indûment élogieux, soit indiscrètement condamnateur. L'autobiographie n'est possible, poursuit le poète toscan, que lorsqu'elle est consacrée à l'édification d'autrui ou qu'elle répond à une accusation. Ainsi, dans L'Enfer,

\footnotetext{
${ }^{9}$ Siegfried Wenzel, «The Pilgrimage of Life as a Late Medieval Genre », Medieval Studies, 35, 1973, p. 370388.

${ }^{10}$ Cette remarque avait déjà été formulée par Maupeu à propos de L'Advision Cristine (Pèlerins de vie humaine, op. cit., p. 425-427). Berriot-Salvadore propose ailleurs («La Mutation de Fortune de Clément Marot», Clément Marot. À propos de "L'Adolescence clémentine», 1996, p. 89-101) un rapprochement entre le Livre de la Mutation de Fortune de Christine de Pizan et cette allégorie de Marot, mais son parallèle est thématique (il porte sur le cadre du pèlerinage et le rôle de Fortune) et non structurel (entre autobiographie et allégorie narrative) comme nous souhaiterions ici procéder.

${ }^{11}$ Jean Frappier, «Sur quelques emprunts de Clément Marot à Jean Lemaire de Belges », Mélanges Edmond Huguet, 1940, p. 161-176.

12 Jean Lemaire de Belges, Les Épîtres de l'Amant vert, éd. Jean Frappier, 1948.

${ }^{13} \mathrm{P}$. Maupeu, Pèlerins de vie humaine, op. cit., p. 23. Voir aussi plus loin, à propos de L'Advision Cristine : « À l'instar de ses prédécesseurs médiévaux, Abélard, Deguileville ou pour remonter plus loin Boèce, l'autobiographie se met en scène comme la réfutation circonstanciée d'une accusation » (p. 456).
} 
Clément commence son plaidoyer en rappelant son prénom, dont il remotive l'étymologie pour établir son innocence : «Car tu es rude, \& mon nom est Clément ${ }^{14}$. La défense de Marot face à Rhadamantus passe ainsi par un système d'oppositions binaires : de même que le prénom du poète est «contraire » à l'esprit de Rhadamantus, de même l'évocation édénique de son Midi natal contraste fortement avec la peinture de l'enfer du monde judiciaire. Que ce soit à travers des images topiques du locus amœnus et fertile (« Parquoy la terre avec honneur s'y vest / De mille fruicts, de mainte fleur, \& plante $\rangle^{15}$, ou encore par l'intertexte lemairien, la description du Quercy est peu référentielle : l'éclat et la simplicité attendue de ces images sont de meilleures armes que la seule vérité dans le discours judiciaire de Clément.

Mais le poète défend aussi sa cause auprès d'un autre juge : François $\mathrm{I}^{\mathrm{er}}$, amateur de poésie, dont il sollicite la protection, qu'elle soit immédiate (il espère qu'il le fera sortir de prison une fois que lui-même sera libéré des geôles de Madrid ${ }^{16}$ ) ou sur un plus long terme, en tant que poète du roi ${ }^{17}$. Ici, le rôle de guide de Jean Marot dans l'initiation poétique de Clément devient crucial. Étant à la cour de François I ${ }^{\mathrm{er}}$, c'est lui qui y a introduit son élève et l'a initié à son langage et à sa poésie, comme Clément le rappelle :

Là, où depuis me suis tant pourmené,

Que j'oubliay ma langue maternelle,

Et grossement apprins la paternelle

Langue Françoyse es grands Courts estimée $[\ldots]^{18}$.

Le jeu de remotivation d'une expression figée, l'opposition entre «langue maternelle » et «paternelle $\mathrm{e}^{19}$ », ainsi que le rejet au vers suivant du substantif duquel «paternelle» est l'épithète, soulignent le rôle du père dans ce départ. Pourtant, en tant que modèle, il est doublé d'un autre illustre « $\operatorname{Maro}^{20} »$, dont l'évocation sert un compliment à l'égard de François $\mathrm{I}^{\mathrm{er}}$ :

\footnotetext{
${ }^{14}$ C. Marot, L'enfer, OP2, p. 19-33, v. 348. Selon Claude La Charité (" "Couraige et invention" : Marot et le cycle carcéral », Clément Marot "Prince des poëtes françois», 1997, p. 249-268), le prénom que Marot proclame lui sert à opposer son identité et ses valeurs aux rôles de victime et de coupable que ses bourreaux voudraient lui donner.

${ }^{15}$ C. Marot, L'enfer, OP2, v. 380-381.

16 «Et comme CHRIST, les Ames poulseroit / Hors des Enfers, sans t'en laisser une Umbre : / En ton advis, seroys je poinct du nombre? » (ibid., v. 442-444)

${ }^{17}$ Marot s'attend en effet à quitter le service de Marguerite pour celui de François $I^{\text {er }}$ : « quelcque jour viendra / Que la Sœur mesme au Frere me rendra » (ibid., v. 425-426).

${ }^{18}$ Ibid., v. 400-403.

${ }^{19}$ Dont Berthon rappelle le caractère avant tout plaisant dans L'Intention du poète, op. cit., p. 234.

${ }^{20}$ Pour un relevé commenté des occurrences du jeu entre Marot et Maro sous la plume de Clément (Jean n'ayant jamais fait ce rapprochement), voir Florian Preisig, Clément Marot et les métamorphoses de l'auteur à la Renaissance, 2004.
} 
Quant au surnom, aussi vray qu'Evangille,

Il tire à cil du Poëte Vergille,

Jadis cheri de Mecenas à Romme :

Maro s'appelle, \& Marot je me nomme,

Marot je suis, \& Maro ne suis pas $[\ldots]^{21}$.

Il semble que Clément, convoitant un poste à la cour de François ${ }^{\mathrm{er}}$, revendique pour parvenir à ses fins un autre modèle que celui de son père, peut-être trop concurrentiel, car il y travaille déjà, sans pour autant faire complètement oublier le nom de celui qui lui a enseigné la langue poétique. Il donne de son nom une signification presque cratylique : porter ce patronyme, c'est être destiné à embrasser la carrière de poète, à la nuance près que Marot n'est pas Maro - aveu de modestie, mais aussi revendication d'un projet littéraire propre, d'une translatio studii en faveur du français et de François $\mathrm{I}^{\mathrm{er}}$. Dans ces vers, Clément joue aussi de l'homophonie entre les premières personnes des verbes «être " et «suivre », pour établir plaisamment et non sans nuances une filiation entre lui-même et le grand poète latin, mais aussi avec son propre père. Marot (Clément) n'est pas Maro ou Marot (Jean), mais il les suit $^{22}$. De fait, le parcours géographique et chronologique de Cahors à la cour se fait un pèlerinage poétique, posant les jalons de l'accomplissement d'une vocation et la création d'une esthétique personnelle, marquée par l'humilité par rapport au projet des précédents Maro(t), qui n'est toutefois pas renié. Ces éléments apparaissent plus nettement dans l'épître « Au Roy » de La Suite, où cette fois Jean Marot est explicitement présent, mais parle d'une voix d'outre-tombe.

Publiée pour la première fois en 1533 en tête des Deux heureux Voyages de Genes \& Venise de Jean Marot, cette épître «Au Roy » date pourtant de $1527^{23}$. Alors que son père vient de mourir et que Clément prend ses fonctions auprès de François $\mathrm{I}^{\mathrm{er}}$, le jeune poète déplore l'oubli de son inscription dans les états de la cour, si bien qu'il ne peut recevoir ses gages. Il demande dans cette épître à recevoir l'argent correspondant au poste que le roi lui a promis. Par le récit des derniers mots de Jean, Clément entend cette fois souligner sa conformité avec son défunt père, montrer qu'il a suivi ses conseils, et ainsi encourager le roi à appuyer cette continuité sur un plan économique. Mais la prosopopée de Jean s'adresse à un fils qui n'a pas encore pris «le droict chemin du service des Princes $»^{24}$, alors que Clément, en 1527, est

\footnotetext{
${ }^{21}$ L'Enfer, v. 359-363.

${ }^{22}$ La confusion des Maro(t) est encouragée par le fait que la présence ou non du $t$ final de Marot ne peut être entendue dans le discours oral que le poète est censé prononcer devant Rhadamantus, et que de toute façon son « Griffon » (v. 452) « ne sceut oncq bien ortographier / Ce qui servoit à [le] justifier » (v. 459-460).

${ }^{23}$ Voir G. Berthon, L'Intention du poète, op. cit., p. 98-107.

${ }^{24}$ Au Roy, OP1, v. 70.
} 
depuis près de dix ans le poète de la sœur du roi, et qu'il a déjà écrit maintes fois en l'honneur de François $\mathrm{I}^{\mathrm{er}}$. Une fois dénoncé ce «gros mensonge ${ }^{25} »$, il convient d'examiner de quelle manière et à quelle fin la réalité de l'apprentissage poétique de Clément est ainsi transformée. La scène où Jean tient la main droite de son fils pour lui transmettre ses dernières volontés est trop idéale pour être lue comme une véritable retranscription ${ }^{26}$. En tant que «bon Vieillard» prodiguant une foule de conseils désintéressés ${ }^{27}$, Jean se rapproche des figures de guides bienveillants peuplant les pèlerinages allégoriques (et qui s'opposent généralement aux guides malveillants que rencontrent d'abord les pèlerins, telle Sensualité chez Saint-Gelais). Ces guides sont souvent des ermites ou des vieillards, comme par exemple à la fin de la Concorde des deux langages de Lemaire, où « Labeur historiien » est un « bon ancien viellart ${ }^{28}$ » auprès duquel l'écrivain trouve enfin sa place. Chez Clément, avant l'épître XXIV de La Suite, d'autres « bons vieillards » sont apparus et peuvent tous être identifiés sinon à Jean, du moins à une figure de poète, courtisan, et guide du jeune élève : dans la traduction de la première églogue des Bucoliques de Virgile, où Tityre, le poète protégé, est un « Heureux Vieillard ${ }^{29}$ », et dans l'«Epistre du Despourveu » où Bon Espoir, la personnification qui vient conforter l'épistolier, est aussi «Ung bon Vieillard ${ }^{30} »$, qui réapparaîtra dans $L$ 'Eglogue au Roy ${ }^{31}$, en désignant cette fois clairement Jean Marot. Le souvenir du récit de formation allégorique précède et dépasse donc la représentation factuelle du père pour souligner son rôle symbolique de guide, prodiguant soutien moral et conseils de carrière.

Les recommandations énumérées dans la prosopopée du guide spirituel s'accordent parfaitement avec le parcours du jeune poète tel qu'il apparaît dans L'Adolescence clémentine. Les injonctions paternelles se superposent avec l'ordre des premiers poèmes du recueil, faisant de cette suite de pièces liminaires le témoin d'un apprentissage poétique, d'un parcours prenant rétrospectivement sens et menant l'apprenti écrivain jusqu'au « service des Princes ». En effet, le «bon Vieillard» conseille en premier lieu les traductions ${ }^{32}$, exercice auquel s'est prêté Clément avec la Première Églogue de Virgile, Le Jugement de Minos de Lucien (par le truchement d'une traduction de Miélot), Les Tristes vers de Philippe de

\footnotetext{
${ }^{25}$ Expression de François Cornilliat, qui s'intéresse également au sens de cette mise en scène dans "Clément Marot et le "noble Art Poëtique", ou la preuve par le père », dans Quant l'ung amy pour l'autre veille, 2008, p. 195-206.

26 OP1, p. 328-329, v. 39-80.

27 Ibid., p. 329, v. 79.

28 Jean Lemaire de Belges, La Concorde des deux langages, 1947, p. 45, 1. 300.

${ }^{29} O P 1$, p. 24, v. 97 et 108 .

${ }^{30} O P 1$, p. $74-75$, v. $98-99$.

${ }^{31} O P 2$, p. 36, v. 63.

${ }^{32} O P 1$, p. 329, v. 57-59.
} 
Béroalde, et enfin L'oraison contemplative devant le crucifix (à partir de Barthélemy de Loches). Le Temple de Cupido et dans une moindre mesure l'Epistre de Maguelonne, bien que n'étant pas des traductions, sont des réécritures de textes antiques et médiévaux qui témoignent d'un détachement progressif de la traduction en faveur d'une production personnelle. Par ce mouvement, mais aussi en faisant se succéder des auteurs chrétiens aux traductions et imitations à l'antique, Clément se conforme à nouveau aux impératifs formulés par son père :

\footnotetext{
Apres tu peulx de ton invention

Faire quelcque Euvre pour jecter en lumiere :

Dedans lequel en la Feuille premiere

Doibs invocquer le nom du tout puissant $[\ldots]^{33}$.
}

Ensuite, le poète pourra chercher «proffit» et «honneur» en se mettant au service d'un prince $^{34}$, ce qui fait écho, au début de L'Adolescence clémentine, à l'«Epistre du Despourveu » par laquelle Marot entre au service de Marguerite. Il lui destine ensuite les épîtres III et IV décrivant la campagne militaire du Hainaut. Enfin, Jean mourant presse son fils d'adresser au roi une œuvre qui lui assurera sa succession comme poète de cour en titre ${ }^{35}$ : le « bon Vieillard » évoque-t-il ici Le Temple de Cupido, une des premières œuvres originales de Clément présentée au jeune couple royal pour solliciter sa protection, ou bien fait-il référence à la présente épître, composée dans l'urgence d'une erreur administrative menaçant la succession du fils au père ? Cette hésitation remet en lumière le décalage chronologique entre la mort de Jean et les premières œuvres de Clément qu'il présente à l'état de projet seulement, comme si le plus jeune Marot voulait précisément insister sur la dimension parabolique et poétique de cette prosopopée.

En incorporant le souvenir de son père dans la trame des pèlerinages allégoriques, Clément invite son lecteur à lire ce discours moins comme une réminiscence lyrique que comme la mise en scène symbolique d'un parcours poétique au service du roi qui exploite ça et là quelques biographèmes - ce que l'existence d'un père poète rend particulièrement aisée. Comme l'expliquent Christine Reno et Liliane Dulac dans l'introduction du Livre de l'advision Cristine :

\footnotetext{
${ }^{33}$ Ibid., v. 60-63.

${ }^{34}$ Ibid., v. 68, 64-65.

${ }^{35}$ Ibid., v. 73-76.
} 
L'autobiographie n'est donc pas une fin en soi; elle fournit l'occasion et le moyen d'un dépassement exemplaire, qui doit lui-même être replacé dans un mouvement d'ensemble ${ }^{36}$.

Ce mouvement d'ensemble est tout d'abord d'ordre rhétorique : l'épître " Au Roy » est une requête que Clément adresse au souverain pour toucher son salaire. En donnant un sens téléologique aux premières pièces de L'Adolescence clémentine, conçues depuis leur origine pour lui permettre d'entrer au service de François ${ }^{\mathrm{er}}$, Clément justifie sa position à la $\operatorname{cour}^{37}$ : il a été élevé pour servir les princes. C'est un projet de longue date, conforme aux dernières volontés (certes antidatées) d'un défunt apprécié par le roi. Clément donne ainsi un argument doublement poétique : par son contenu (il succède à un père poète qui lui a tout appris) et par sa forme, qui évoque d'autres souvenirs littéraires. En outre, la prosopopée de Jean, lui-même devenu figure exemplaire de sagesse et de bienveillance, dédouane Clément de l'audace d'adresser une requête au roi, ce qui est un élément important dans le cadre d'une demande d'argent, a priori non honorable ${ }^{38}$. La question de la vérité du contenu des propos de Jean vaut donc moins que l'image du parcours poétique et courtisan idéal de Clément qui transparaît et qu'il construit sur le modèle des pèlerinages allégoriques. On songe au Séjour d'Honneur où le poète, d'abord mal guidé par Sensualité, puis chassé de la cour d'Honneur où il montrait trop d'ambition, parvient à l'ermitage d'Entendement : Saint-Gelais s'efforce, par ce récit, de prouver à Charles VIII que, malgré sa jeunesse, il écrit avec le recul d'un homme mûr ayant déjà traversé bien des épreuves et qu'il est désormais apte au service courtisan. Clément valorise de même sa propre initiation.

Mais la prosopopée de Marot revêt également des enjeux esthétiques ${ }^{39}$. Lorsqu'il hérite du poste de Jean à la cour du roi, Clément doit prouver que son talent poétique est à la hauteur de son nom - celui de son géniteur - et que cette succession n'est pas indue. La difficulté (certes accidentelle) à obtenir ses gages est pour Clément le signe qu'il doit justifier non seulement son salaire, mais aussi convaincre de sa légitimité à occuper le poste de son père. Le jeune

\footnotetext{
${ }^{36}$ C. Reno et L. Dulac, 2001, p. XVIII.

${ }^{37}$ Olivia Rosenthal parvenait à une conclusion semblable, sur le rôle de l'épître XXIV comme clé de voûte d'une carrière et d'une œuvre, dans «Clément Marot, une poétique de la requête », Clément Marot « Prince des poëtes françois », 1997, p. 283-299, en analysant l'ordre des épîtres XIV à XXIV de La Suite de 1538.

38 Selon les traités rhétoriques ou épistolographiques de l'époque, comme le Grant et vray art de pleine rhetorique de Fabri ou le De Conscribendis epistolis d'Érasme.

${ }^{39}$ Dimension entrevue par Maupeu à propos du Pèlerinage de Vie humaine de Deguileville (dans sa deuxième rédaction): «L'énoncé autobiographique n'est pas traversé d'un réel qui lui préexisterait: il relève d'une stratégie d'exposition de soi qui s'inscrit dans le cadre général d'une poétique. » (Pèlerins de vie humaine, op. cit., p. 12).
} 
poète doit montrer qu'il est le digne élève de son maître ${ }^{40}$, sans en être la reproduction imparfaite ou servile. Dans le fameux distique «Et ne falloit, Sire, tant seulement, / Qu'effacer Jan, \& escrire Clement ${ }^{41}$, il cherche, pour toucher les gages du poste dont il vient d'hériter, à montrer tout ce qu'il a de commun avec son père, tout en se réservant une part d'originalité, incarnée ici par son prénom ${ }^{42}$. Clément met en scène, par la prosopopée du « bon vieillard », ce qu'il doit à celui que tout le monde considère comme son maître, mais par le décalage chronologique au cœur de ce récit (et de la genèse du poème, écrit en 1527 mais publié en 1533 en tête d'un recueil d'œuvres historiographiques du père) il revendique de ne pas en être la copie conforme, d'avoir une légitimité propre pour occuper la fonction offerte par François $\mathrm{I}^{\mathrm{er}}$. François Cornilliat résume ainsi le geste de Clément :

[...] l'enjeu majeur de l'épître n'est pas seulement d'assimiler symboliquement l'art du père à l'art du fils en récrivant l'histoire de la filiation, mais aussi de marquer implicitement leur différence rhétorique réelle, telle qu'elle se manifeste, en particulier, lorsqu'on s'adresse au Roy: l'un raconte la grande histoire et l'autre non; et c'est celui qui ne la raconte plus qui a les moyens de repeindre la petite histoire de son père $[\ldots]^{43}$.

Dans cette épître, Clément rend significatifs et exemplaires les faits et accidents de la succession à Jean pour, d'une part, appuyer une requête d'argent et, d'autre part, arrivant à la cour de François ${ }^{\text {er }}$ sans toutefois vouloir paraître arriviste, poser les bases d'une poétique qui, tout en refusant de rompre brutalement avec celle de celui qu'il n'ose présenter comme son maître, mais plutôt comme un guide, n'en constitue pas pour autant une reproduction disciplinée. L'Eglogue au Roy, qui développe le récit de l'enfance de Clément auprès de Jean, approfondit ce mélange de dette et de transformation.

Écrite en 1539, cette églogue mêle fiction pastorale et autobiographie. Sous les traits de Robin, Clément raconte comment son père Janot lui a enseigné la poésie pour qu'il devienne à son tour un berger-poète au service du grand dieu Pan. Mais le fils n'a pas pris le même soin que son père à mettre des richesses de côté, si bien qu'il se retrouve démuni quand la vieillesse arrive : c'est pourquoi il finit son églogue en demandant à Pan/François ${ }^{\text {er }}$ de lui octroyer une maison, qui préservera son troupeau du froid et lui permettra de chanter à

\footnotetext{
${ }^{40}$ Alors même que Jean n'apparaît pas, alors, comme un véritable précepteur, offrant des leçons techniques : ce rôle semble plutôt dévolu à Lemaire, maître ès coupes féminines dans l'épître liminaire de L'Adolescence clémentine (OP1, p. 18).

${ }^{41}$ OP1, p. 328, v. 21-22.

${ }^{42}$ Cela nuance les analyses de Gérard Defaux dans “"Effacer Jean, \& escrire Clément” : une douloureuse (et double) affaire de succession », La Génération Marot, 1997, p. 81-112, et de Jean-Charles Monferran dans "Père et fils dans l'Adolescence Clémentine: "Effacer Jean et escrire Clement"”, La Poétique des passions à la Renaissance, 2001, p. 125-140.

${ }^{43}$ F. Cornilliat, « Clément Marot et le "noble Art Poëtique”, ou la preuve par le père », art. cité, p. 205.
} 
nouveau. Même si ce poème n'est pas proprement allégorique, la narration bucolique et les liens qu'elle entretient avec la biographie du poète permettent d'analyser ce récit de formation reconstitué comme l'élément d'une rhétorique argumentative ou l'incarnation d'un discours exposant la poétique de Clément ${ }^{44}$. La pastorale possède une longue tradition depuis Virgile, auteur que Marot réécrit ici abondamment, et s'accommode volontiers de certains biographèmes. Il est néanmoins établi que, dans cette églogue, Clément emprunte aussi ses mots à Lemaire pour décrire sa jeunesse ${ }^{45}$. Ainsi, ses activités de plein air sont de toute évidence inventées d'après le roman de l'enfance de Pâris dans les Illustrations ${ }^{46}$. Cette mise en vers à peine recomposée du récit lemairien - parmi d'autres intertextes, et parmi la foule de personnages topiques de la pastorale ou des allégories morales tel que le «bon vieillard ${ }^{47}$ - révèle une nouvelle fois la façon dont le poète interprète les aléas de son existence et de sa carrière (départ du Quercy, apprentissage des lettres avec un père poète, maladie, précarité, exil) à l'aune de ses modèles littéraires, afin d'en faire un parcours significatif, un pèlerinage édifiant ${ }^{48}$, mais dans un domaine plus poétique que moral: contrairement au grand modèle du Pèlerinage de Vie humaine et à l'instar des allégories de Christine de Pizan, le poète de Cahors décrit moins sa naissance à la vertu qu'à l'écriture.

Résumée à sa plus brève propositio, l'Eglogue au Roy est une requête adressée à François I $^{\mathrm{er}}$ portant sur une maison hors de la cour, afin que le poète puisse y abriter ses enfants ${ }^{49}$. Pour appuyer cette demande, Clément rappelle qu'il est le fils de Jean, serviteur dévoué de François $\mathrm{I}^{\mathrm{er}}$ jusqu'à sa mort, lequel a déjà été récompensé par le roi : le fils sollicite la même faveur. En même temps, il met à distance cette succession poétique en précisant, sous forme d'anteoccupatio, que sa négligence ne lui a pas permis d'hériter des biens de son père ou de

\footnotetext{
${ }^{44}$ Le mythe allégorique et la pastorale se rencontraient déjà dans la fiction autobiographique de L'Enfer : après avoir désigné la famille royale au moyen des noms de divinités ayant leur fonction (Jupiter, Pallas, Cybèle, v. 316-323), Marot évoque d'autres personnages mythologiques sans que l'on puisse les rattacher à des personnages éminents en vertu d'une lecture à clefs (v. 326-330). Avec Pan et son épouse Églé (v. 331-332) l'énumération des soutiens de Marot se fait pastorale et inclut Galathée et Tityre (v. 334-336), venus des Bucoliques de Virgile.

${ }^{45}$ Sur ces intertextes, voir Jean Bayet, « La source principale de l'églogue de Clément Marot “Au Roy soubs les noms de Pan et Robin" ", RHLF, 34, 1927, p. 567-571; Gustave Charlier, "Sur l'enfance de Marot », ibid.,, p. 426-428; J. Frappier, «Sur quelques emprunts de Clément Marot à Jean Lemaire de Belges », art. cité, ainsi que le complément d'Adeline Desbois-Ientile dans L'histoire écoutée aux portes de la mythologie : l'écriture du mythe troyen autour des Illustrations de Lemaire de Belges, 2015, p. 481.

${ }^{46}$ Voir les vers 15-39 de l' Eglogue au roy, soubs les noms de Pan, \& Robin, OP2, p. 34-41, et la page 151 des Illustrations (dans Jean Lemaire de Belges, Euvres, 1969, vol. 1).

${ }^{47}$ Eglogue au Roy, v. 63.

${ }^{48}$ Le motif du pèlerinage apparait aux vers 171-174 : «Le blond Phebus, qui me voyt, \& regarde, / Si l'espesseur de ce boys ne l'engarde : / Et qui m'a veut traverser maint Rochier, / Et maint torrent pour de toy approcher. »

${ }^{49}$ Defaux hésite sur la nature rhétorique de ce texte, dans lequel il voit peut-être aussi un remerciement, une fois que le don de la maison du Clos-Bruneau a été accordé (OP2, notes p. 813). Nous pensons que le remerciement est anticipé à la fin de la requête de Clément.
} 
les faire fructifier, d'où sa précarité actuelle. De nouveau, l'autobiographie de Clément, et en particulier l'évocation des enseignements poétiques de son père, est constituée à des fins rhétoriques, et une fois encore cette argumentation est dépassée par une réflexion sur le métier de poète, notamment de cour, que Clément formule avec et contre l'exemple paternel.

Durant « le printemps de [sa] jeunesse folle ${ }^{50}$, le fils Marot s'efforce de montrer ce que son choix d'une carrière poétique doit aux efforts paternels, tout en présentant discrètement sa vision personnelle du rôle du poète et de son art. Tout d'abord, il reprend la genèse de sa vocation, en plaçant ses dispositions naturelles avant le travail du « bon Janot» :

\footnotetext{
Si ne sçauroys bien dire, ne penser,

Qui m'enseigna si tost d'y commencer,

Ou la nature aux Muses inclinée,

Ou ma Fortune, en cela destinée

A te servir: si ce ne fut l'ung d'eulx,

Je suis certain, que ce furent touts deux ${ }^{51}$.
}

La poésie est en partie naturelle à Clément, une nature qu'il partage avec son géniteur. Mais elle repose aussi sur les leçons essentielles de ce dernier. Il complète ainsi les modalités de son apprentissage autour de la tripartition natura, doctrina et usus :

\footnotetext{
Et me souvient, que bien souvent aux Festes

En regardant de loing paistre noz bestes,

Il me souloit une leçon donner,

Pour doulcement la Musette entonner,

Ou à dicter quelcque chanson ruralle

Pour la chanter en la mode pastoralle.

Aussi le soir, que les trouppeaulx epars

Estoyent serrés, \& remis en leurs parcs,

Le bon vieillard apres moy travailloit,

Et à la lampe assez tard me veilloit $[\ldots]^{52}$.
}

Jean Marot est présenté comme un maître de poésie, le premier que Clément connaît au moment où sa vocation apparaît: pour attendue qu'elle soit, cette image est inédite dans le corpus des mentions de son père par Clément, qui le montrait plus comme un soutien moral ou un guide de carrière que comme un véritable précepteur. Ses fréquentes leçons de poésie relèvent de l'apprentissage de la doctrina. Le dévouement de Jean envers son fils n'a d'égal que celui dont le poète fait preuve envers son roi, un zèle qui réapparaît au terme de la formation de Clément, lorsqu'il dévoile le but de son étude :

\footnotetext{
${ }^{50}$ Eglogue au roy, v. 15.

${ }^{51}$ Ibid., v. 43-48.

${ }^{52}$ Ibid., v. 55-64.
} 
Certes c'estoit affin qu'en l'imitant,

A l'advenir je chantasse le los

De toy (ô Pan) qui augmentas son clos $[\ldots]^{53}$.

L'imitation est le meilleur moyen pour un poète d'apprendre en s'exerçant : c'est l'usus tant valorisé dans les arts poétiques du $\mathrm{XVI}^{\mathrm{e}}$ siècle, de Sébillet à Peletier. Avec la pratique, Clément indique aussi les fins de la poésie: louer les grands pour leurs bienfaits, à commencer par Pan/François $\mathrm{I}^{\mathrm{er}}$, qui a déjà aidé le père dans son propre «Yver ${ }^{54}$. Jean exerçait avec dévouement ses fonctions de poète de cour ; puisque c'est lui qui a enseigné technique et usage de la poésie à Clément, ce dernier suggère qu'il ne peut qu'en faire autant. Par conséquent, il est en droit de demander des rétributions semblables. Le syllogisme est clos et l'argument, qui s'appuie sur le bon service du père et maître, pourrait suffire.

Clément oppose pourtant son peu de soin à la sollicitude paternelle, lorsque celui-ci lui montre l'étendue d'un domaine que ses vers lui ont obtenu :

\footnotetext{
Ainsi soigneux de mon bien me parloit Le bon Janot, \& il ne m'en challoit :

Car soucy lors n'avoit en mon courage

D'aulcun bestail, ne d'aulcun pasturage ${ }^{55}$.
}

Le souci du «bien» de son fils est une constante du portrait de Jean par Clément, plus souvent père que poète, guide que véritable maître. Ces indications matérielles, quoiqu'essentielles à la présente demande de Clément, occultent en outre le contenu exact du programme d'apprentissage poétique dispensé par Jean. Enfin, ces vers, qui mettent un terme à la partie de l'églogue consacrée au «printemps» poétique de Clément, marquent une rupture entre le père et le fils qui, si elle est traitée sur le mode d'une simple négligence de la part de Clément, n'en est pas moins capitale pour comprendre la petitio adressée au roi ainsi que la façon dont il conçoit personnellement le métier de poète de cour ${ }^{56}$. En effet, pour Jean, la poésie appelle naturellement sa rémunération en «bien»: comme dans l'épître « $\mathrm{Au}$ Roy », Jean conseille à son fils de chanter la gloire de Pan/François I ${ }^{\text {er }}$ pour obtenir de lui

\footnotetext{
${ }^{53}$ Ibid., v. 74-76.

${ }^{54}$ Ibid., v. 222.

55 Ibid., v. 103-106.

${ }^{56}$ De même, dans L'Enfer, Clément n'avait que « grossement » appris la « langue paternelle » de la cour et de la poésie. D'un point de vue rhétorique, le poète fait de l'humilité de sa poésie moins une recherche qu'un défaut d'apprentissage, ce qui est une captatio benevolentice conventionnelle. D'un point de vue esthétique, le fils annonce déjà qu'il ne suivra qu'imparfaitement les conseils de son maître : reconnaissant son enseignement, il n'en est toutefois pas la reproduction.
} 
protection, à commencer par une maison «plaisante », qu'il a obtenue et qui est précisément 1'objet de la demande de Clément ${ }^{57}$. Si ce dernier avait bien écouté son maître, il n'aurait qu'à chanter à son tour les louanges de Pan pour obtenir ce qu'il désire. Mais Clément est un disciple peu attentif, et il suggère ainsi une rupture plus profonde entre sa conception de la poésie de cour et celle de son père. Il refuse de faire l'éloge du roi en vue d'obtenir un don. S'il lui est accordé, celui-ci devra l'être en rémunération d'un autre don que seul un poète est capable de faire, celui du «plaisir » :

\author{
Et penses tu (ô Pan Dieu debonnaire) \\ Que 1'exercice, \& labeur ordinaire, \\ Que pour sonner du Flageolet je pris, \\ Feust seullement pour emporter le pris? \\ Non : mais affin que si bien j'en apprinsse, \\ Que toy, qui es des Pastoureaulx le Prince, \\ Prinsses plaisir à mon chant escouter $[\ldots]^{58}$.
}

$\mathrm{Au}$ « bien », au «pris » tant convoités par le père s'oppose le «plaisir » seul que Clément veut offrir à son protecteur ${ }^{59}$. À ces valeurs opposées s'ajoute une autre distinction : celle du travail quotidien du poète («labeur ordinaire») et de la réception libre du poème par le protecteur, sous forme de " grâce ».

La poésie de Clément repose donc sur d'autres valeurs que celles du travail ou de la nécessité financière. De ce point de vue, le monde pastoral représenté dans cette églogue ne constitue pas tant une métaphore de la cour (susceptible d'être résolue au moyen de clés) qu'une dimension poétique de celle-ci : Marot présente à son monarque un espace bucolique dans le jeu de la cour, mais à l'écart du pouvoir. L'évocation, même voilée, de l'enfance de Pâris, lorsqu'il n'était encore qu'un prince pastoral et avant qu'il ne (re)devienne fils de Priam, puissant et belliqueux, s'apparente à une invitation adressée à François ${ }^{\text {er }}$ à délaisser les affaires du pouvoir et de l'argent, et à redevenir, le temps d'un poème, un prince champêtre, comme son ancêtre des Illustrations. Le charme du chant marotique tient dans cette invitation à prendre de la distance, sans verser pour autant dans la rupture que supposerait une représentation purement symbolique. Cette définition d'un art poétique à l'écart des affaires et hiérarchies de la cour, qui repose sur un plaisir partagé, ne va toutefois pas sans arrièrepensées : dans ce poème de requête, Marot rappelle le plaisir qu'il a procuré au roi, ce don qu'il lui a fait, en échange duquel le roi peut librement lui accorder un autre don. En racontant

\footnotetext{
${ }^{57}$ Eglogue au roy, v. 73-92.

${ }^{58}$ Ibid., v. 159-165.

${ }^{59}$ Sur cet aspect de la poétique de Clément, voir l'étude de la « Petite Epistre au Roy » de F. Cornilliat dans «Or ne mens », 1994, p. 330-338.
} 
sa vie de berger-élève dans une églogue légère, Marot s'efforce de nouveau d'agrémenter les passe-temps de son roi qui peut, s'il le souhaite, le remercier d'une faveur royale.

$\mathrm{Au}$ croisement de la genèse du poète et de la poésie, la figure exemplaire et symbolique de Jean Marot constitue un argument à la requête de Clément et alimente une réflexion comparatiste sur l'art poétique marotique. Si elle invite dans un premier temps François $I^{\text {er }}$ à la bienveillance en lui rappelant l'œuvre dévouée du père et l'héritage du fils, elle sert dans un second temps de repoussoir à Clément, écrivain désormais confirmé qui adopte une autre poétique. Le système de grâces réciproques remplace désormais celui du travail salarié qui fondait la poésie de son maître - reconnu en tant que tel au moment où le disciple affirme son autonomie - et qui se cristallise dans la nonchalance de Clément. Il convient donc de distinguer, du père au fils Marot, l'héritage du talent poétique, inné et acquis ; l'héritage des biens matériels, entravé par la nonchalance du jeune poète ; et enfin l'héritage du métier de poète de cour, que Clément transforme de l'intérieur, faisant de sa négligence matérielle un hommage au père, guide et maître et une confiance dans la grâce que le prince pourra lui accorder.

En somme, les évocations par Clément de son père sont bien plus motivées par les nécessités rhétoriques de la défense ou de la requête que par l'expression lyrique. C'est une des raisons pour lesquelles Clément organise les éléments du récit de son apprentissage auprès de Jean et de son arrivée à la cour de François $\mathrm{I}^{\mathrm{er}}$ selon le genre du pèlerinage allégorique, tel qu'il a pu le lire dans les récits d'écrivains courtisans qui le précèdent : la dimension exemplaire qu'il confère à ses biographèmes en augmente l'efficacité rhétorique. Mais le pèlerinage de Clément, de l'idyllique Midi aux vicissitudes de la vie parisienne et courtisane, raconté dans les portraits en actes et en paroles de celui qui est tantôt guide symbolique, tantôt précepteur studieux, a surtout des fins poétiques, qui varient selon que Clément désire se démarquer de son père travaillant déjà à la cour de François $I^{\mathrm{er}}$, souligner une continuité et un talent partagé lorsque celui-ci s'éteint, ou encore renouveler sans rupture les fondements de la poésie de cour. La présentation pseudo autobiographique de cette poétique n'est du reste pas innocente : pour paraphraser une dernière fois $\mathrm{Maupeu}^{60}$, en inscrivant ce manifeste dans l'épaisseur temporelle d'une expérience personnelle et familiale, Clément accorde un statut heuristique à sa poétique, une valeur à sa simplicité.

Ellen Delvallée

Rutgers University / Université Grenoble-Alpes

\footnotetext{
${ }^{60}$ Dans son analyse de L'Advision Cristine (Pèlerins de vie humaine, op. cit., p. 464).
} 


\section{BIBLIOGRAPHIE}

BAYET, Jean, «La source principale de l'églogue de Clément Marot “Au Roy soubs les noms de Pan et Robin”’», RHLF, 34, oct-déc 1927, p. 567-571.

BERRIOT-SALVADORE, Evelyne, «Le "pèlerinage de vie humaine” de Clément Marot », Revue de littératures françaises et comparée, 7, 1996, p. 47-53.

BERRIOT-SALVADORE, Evelyne, «La Mutation de Fortune de Clément Marot», dans James DAUPHINÉ et Paul MIRONNEAU (éd.), Clément Marot. À propos de L'Adolescence clémentine. Acte des quatrièmes journées du Centre Jacques de Laprade (29 et 30 novembre 1996), Biarritz, J\&D Éditions, 1996, p. 89-101.

BERTHON, Guillaume, L'Intention du poète. Clément Marot « autheur », Paris, Classiques Garnier, 2014.

BERTHON, Guillaume, "Clément Marot et la tentation biographique », Philippe DESAN (dir.), Cahiers parisiens / Parisian notebooks, Paris, University of Chicago Center in Paris, 4, 2008, p. 541-551.

BOURGEOIS, Françoise, LE BRIZ-ORGEUR, Stéphanie, BIESHEUVEL, Ingrid et DOR, Juliette, section «Réécritures et postérité du modèle des Pèlerinages », dans Frédéric DUVAL et Fabienne POMEL (éd.), Guillaume de Digulleville. Les Pèlerinages allégoriques, Presses Universitaires de Rennes, 2008, p. 351-423.

CHARLIER, Gustave, «Sur l'enfance de Marot », RHLF, XXXIV, juillet-sept 1927, p. 426428.

CHRISTINE de PIZAN, Livre de l'advision Cristine, Paris, Champion, 2001 [Christine Reno et Liliane Dulac (éd.)].

CORNILLIAT, François, «Clément Marot et le "noble Art Poëtique”, ou la preuve par le père », dans Tania VAN HEMELRYCK et Maria COLOMBO TIMELLI (éd.), Quant l'ung amy pour l'autre veille. Mélanges de moyen français offerts à Claude Thiry, Turnhout, 
Brepols, 2008, p. 195-206, repris dans Sujet caduc, noble sujet : la poésie de la Renaissance et le choix de ses arguments ", Genève, Droz, 2009, p. 960-965.

CORNILLIAT, François, "Or ne mens ». Couleurs de l'éloge et du blâme chez les " Grands Rhétoriqueurs », Paris, Champion, 1994.

DEFAUX, Gérard, «"Effacer Jean, \& escrire Clément” : une douloureuse (et double) affaire de succession », dans Gérard DEFAUX (éd.), La Génération Marot. Poètes français et néolatins (1515-1550). Actes du colloque international de Baltimore - 5-7 décembre 1996, Paris, Champion, 1997, p. 81-112.

DEFAUX, Gérard, «Clément Marot : poésie, autobiographie et roman », dans Raymond C. LA CHARITÉ (éd.), Writing the Renaissance. Essays on Sixteenth-Century French Literature in Honor of Floyd Gray, Lexington (Kentucky), French Forum Publishers, 1992, p. 79-91.

DESBOIS-IENTILE, Adeline, L'histoire écoutée aux portes de la mythologie : l'écriture du mythe troyen autour des Illustrations de Lemaire de Belges, thèse de doctorat réalisée sous la direction de Mireille Huchon, Paris Sorbonne, 2015.

FRAPPIER, Jean, «Sur quelques emprunts de Clément Marot à Jean Lemaire de Belges », Mélanges de philologie et d'histoire littéraire offerts à Edmond Huguet, Paris, Boivin, 1940, p. 161-176.

LA CHARITÉ, Claude, " “Couraige et invention” : Marot et le cycle carcéral », dans Gérard DEFAUX et Michel SIMONIN (éd.), Clément Marot «Prince des poëtes françois », 14961996. Actes du Colloque international de Cahors en Quercy, 21-25 mai 1996, Paris, Champion, 1997, p. 249-268.

LEMAIRE DE BELGES, Jean, Euvres, Genève, Slatkine Reprints, 1969, 4 vol. [JeanAuguste Stecher (éd.)].

LEMAIRE DE BELGES, Jean, Les Épîtres de l'Amant vert, Genève, Droz, 1948 [Jean Frappier (éd.)].

LEMAIRE DE BELGES, Jean, La Concorde des deux langages, Paris, Droz, 1947 [Jean Frappier (éd.)]. 
MAROT, Clément, Euvres poétiques, Paris, Bordas, (Classiques Garnier), 2 tomes, 1990 et 1993 [Gérard Defaux (éd.)].

MAUPEU, Philippe, Pèlerins de vie humaine. Autobiographie et allégorie narrative, de Guillaume de Deguileville à Octovien de Saint-Gelais, Paris, Champion, 2009.

MONFERRAN, Jean-Charles, «Père et fils dans l'Adolescence Clémentine : “Effacer Jean et escrire Clement" », dans François LECERCLE et Simone PERRIER (éd.), La Poétique des passions à la Renaissance. Mélanges offerts à François Charpentier, Paris, Champion, 2001, p. $125-140$.

PREISIG, Florian, Clément Marot et les métamorphoses de l'auteur à la Renaissance, Genève, Droz, 2004.

ROSENTHAL, Olivia, «Clément Marot, une poétique de la requête », dans Clément Marot " Prince des poëtes françois », op. cit., p. 283-299.

SAINT-GELAIS, Octovien, Séjour d'Honneur, Genève, Droz,2002 [Frédéric Duval (éd.)].

VEYSSEYRE, Géraldine avec l'assistance de DOBRINSKY, Julie et de FRINGER, Émilie, «Liste des manuscrits des trois Pèlerinages», dans Guillaume de Digulleville. Les Pèlerinages allégoriques, op. cit., 2008, p. 425-453.

WENZEL, Siegfried, «The Pilgrimage of Life as a Late Medieval Genre », Medieval Studies, 35 , 1973, p. $370-388$. 